\title{
Numerical study of the Time-of-Flight Pulsed Ultrasonic Polar Scan for the determination of the full elasticity tensor of orthotropic plates
}

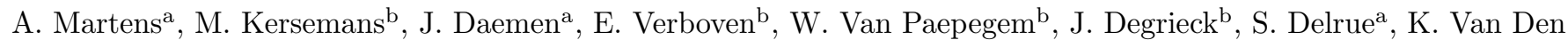 \\ Abeele $^{\mathrm{a}}$ \\ ${ }^{a}$ Wave Propagation and Signal Processing (WPSP), Department of Physics, KU Leuven - Kulak, 8500 Kortrijk, Belgium \\ ${ }^{b}$ Department of Materials, Textiles and Chemical Engineering, Ghent University, Technologiepark-Zwijnaarde 903, 9052 Zwijnaarde, \\ Belgium
}

\begin{abstract}
A novel approach is presented for the ultrasonic determination of the elastic constants in plate-like structures of an orthotropic material (e.g. composites) using a time-of-flight version of the pulsed ultrasonic polar scan (TOF P-UPS). A forward numerical model of the TOF P-UPS is coupled to an inversion algorithm, based on the genetic optimization principle, targeting the determination of the orthotropic elastic parameters, and the quality of the inversion is demonstrated for synthetic data representative for composites. The advantage of the new approach is that the presented TOF P-UPS inversion method does not require a priori knowledge about the symmetry class of the material, nor about the orientation of the main axes of symmetry. Furthermore, the TOF P-UPS inversion method yields an accurate characterization of the orthotropic elasticity tensor, even when applied to composite plates with small frequency-thickness ratios in which the traditional bulk wave approaches no longer hold. Finally, the robustness of the TOF P-UPS inversion method is demonstrated for noisy data by evaluating the results for a range of signal-to-noise ratios.
\end{abstract}

Keywords: Ultrasonic Polar Scan, Time-of-Flight, NDT, characterization

\section{Introduction}

The last few decades experienced an exponential increase in the use of composite materials, such as carbon or glass fiber reinforced plastics (CFRP - GFRP). These composites typically have a low weight to high stiffness ratio, making them particularly interesting for application in aerospace structures and, more recently, in primary, load-bearing components. On the one hand, proper selection of the orientation of the reinforcement fibers leads to a directional mechanical response which may be tuned for a specific functionality, e.g. high stiffness in one direction. On the other hand, however, such a mechanical anisotropy makes it challenging to characterize composites in a straightforward manner. Though, in designing composite components it is of crucial importance to have knowledge on the stiffness tensor in order to assure the component's functionality and to maintain its structural integrity. In the past, several non-destructive characterization techniques have been proposed and developed to obtain information about the elastic material properties on the basis of ultrasound [1-6], in which most characterization techniques simply employ bulk wave concepts. Typically, these techniques rely on the Time-of-Flight (TOF) of an ultrasound pulse, from which the wave velocity can

\footnotetext{
Email address: arvid.martens@kuleuven-kulak.be (A. Martens)
}

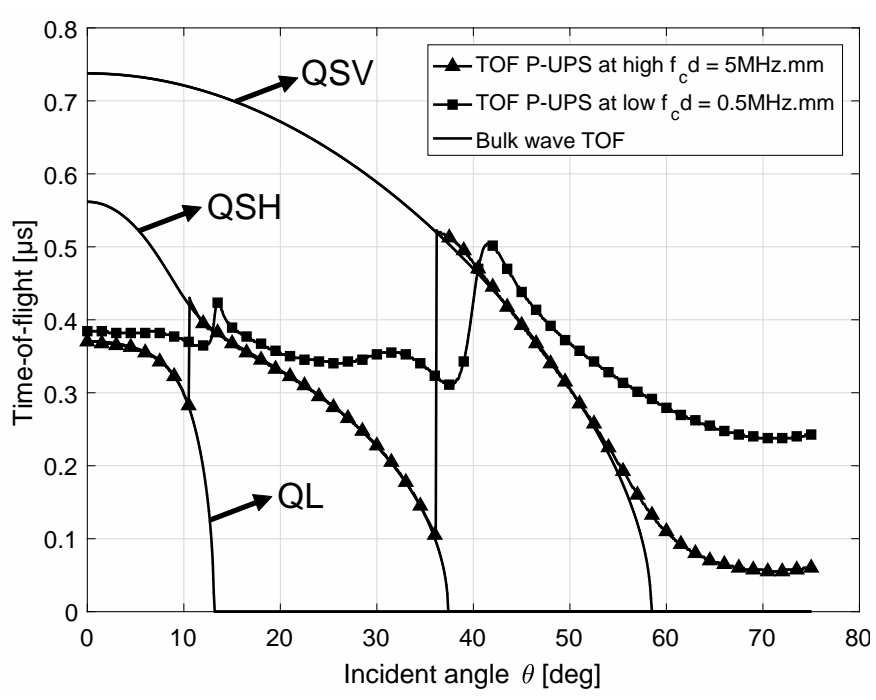

Figure 1: Cross-section (at azimuthal angle $\phi=45^{\circ}$ ) of the TOF P-UPS data for an artificial orthotropic material (cfr. column 3 of table 1 at different $f_{c} d$ values (triangle and square curves). The TOF results for bulk wave propagation (solid curve) for the same azimuthal angle are superimposed.

be determined, which is on its turn related to the mechanical stiffness of the investigated medium [1 8]. Although, these methods have proven their effectiveness, they also suffer from a number of drawbacks which are inherent to the use of bulk waves. 
First of all, ultrasonic bulk waves only exist in infinite media. The use of ultrasonic TOF measurements, however, requires a through transmission phenomenon, and, as such, it can only be applied to samples with finite thickness. In reality, composites are often manufactured and used in the form of a laminate with a thin section. Hence, it is crucial for bulk wave based methods to adjust and select an appropriate wavelength, and thus frequency of the ultrasonic wave, in order to consider and justify the investigated sample as an 'infinite' medium. The importance of selecting the right center frequency $\left(f_{c}\right)$ is explicitly demonstrated in the simulation results shown in figure 1 for a homogeneous unidirectional composite (with elastic constants listed in column 3 of table 1), immersed in water, with thickness $d=0.5 \mathrm{~mm}$. The solid line represents the TOF, associated with bulk wave propagation in the plate sample, as a function of the incident angle $\theta$, for an azimuthal (in-plane) angle of $45^{\circ}$ with respect to the principal symmetry axis ( $x_{1}$-direction). According to the Snell-Descartes law, critical refraction occurs at particular incident angles. The condition for critical refraction can be easily identified in figure 1 by those angles where the individual bulk waves (QL for quasi-longitudinal, QSH for quasi-shear horizontal and QSV for quasi-shear vertical) are cut off. The triangle line corresponds to the TOF data calculated for the transmission of an ultrasonic pulse with center frequency $f_{c}=10 \mathrm{MHz}$, resulting in a frequency-thickness value $f_{c} d=5 \mathrm{MHz} . \mathrm{mm}$. It can be readily observed that the TOF of the pulse with $f_{c} d=5$ MHz.mm $(\lambda \ll d)$ more or less corresponds to the TOF associated with bulk wave propagation. The sharp jumps in the triangle line can be associated to changes in the dominant wave mode in the plate, i.e. a transition from quasilongitudinal to horizontal quasi-transverse polarization at the first jump, and from horizontal quasi-transverse to vertical quasi-transverse polarization at the second jump [9]. Lowering the center frequency of the ultrasonic pulse to $f_{c}=1 \mathrm{MHz}$, yields a frequency-thickness value $f_{c} d=0.5$ MHz.mm, and results in a TOF prediction corresponding to the square curve in figure 1 . In this case, the TOF associated to the $1 \mathrm{MHz}$ pulse deviates substantially from the bulk wave solution. Consequently, the use of bulk wave based inversion methods for the above case with $f_{c} d=0.5 \mathrm{MHz} . \mathrm{mm}$ will definitely lead to erroneous results. Hence, bulk wave based methods set a lower limit on the frequencies to be used. On the other hand, there is also an upper limit for the frequency to be considered in order to have a sufficiently long wavelength to meet the homogenization condition and to avoid internal scattering and diffraction phenomena in inhomogeneous media like composites. Moreover, as high frequency ultrasonic waves are accompanied with a correspondingly high energy loss due to the presence of material damping, the use of a too high excitation frequency will eventually lead to a situation in which no energy at all can be transmitted through the plate, making TOF measurements impossible. The combination of these limits results in a rather narrow fre-
Table 1: Material parameters considered during the generation of the forward simulations.

\begin{tabular}{ccc}
\hline & Aluminum & C/E Composite \\
\hline$C_{11}[\mathrm{GPa}]$ & 110.50 & 122.73 \\
$C_{12}=C_{13}[\mathrm{GPa}]$ & 58.50 & 6.57 \\
$C_{22}=C_{33}[\mathrm{GPa}]$ & 110.50 & 13.47 \\
$C_{23}[\mathrm{GPa}]$ & 58.50 & 6.55 \\
$C_{44}[\mathrm{GPa}]$ & 26.00 & 3.40 \\
$C_{55}[\mathrm{GPa}]$ & 26.00 & 5.86 \\
$C_{66}[\mathrm{GPa}]$ & 26.00 & 6.25 \\
$\rho\left[\mathrm{kg} / \mathrm{m}^{3}\right]$ & 2700 & 1528 \\
\hline
\end{tabular}

quency band where characterization techniques based on bulk-wave approximation can be considered to be valid.

As a second drawback we mention that many bulk wave approximations neglect phase changes of the transverse bulk waves at the interfaces of a liquid/solid/liquid structure 10. These phase changes are induced at the critical angles where a particular wave mode becomes evanescent, and result in a complex valued wave number, which consequently leads to complex valued transmission and reflection coefficients. Obviously, these complex valued scattering coefficients produce a phase that is different from zero (figure 2). Although this phase change $(\Psi(\theta))$ is independent of both frequency and sample thickness, it does induce a time-delay for certain input signals. For instance, sinusoidal input signals (constant frequency $f_{0}$ ) experience a time-delay, when passing a fluid-solid interface, given by 10:

$\Delta t=\frac{\Psi(\theta)}{2 \pi f_{0}}$

To account for this, several authors have extended the traditional bulk wave characterization technique with an

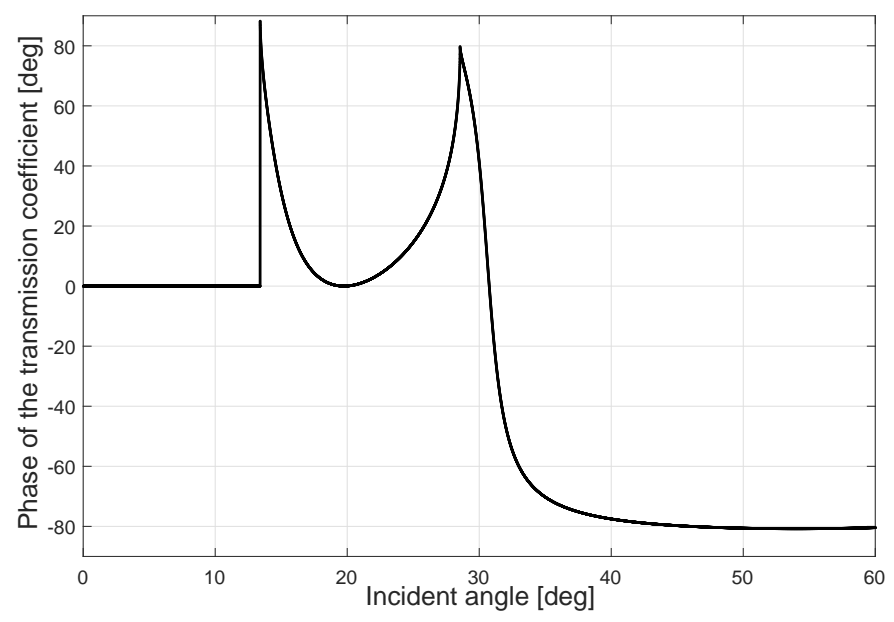

Figure 2: Numerically computed phase of the bulk wave transmission coefficient as a function of incident angle for an aluminum (cfr. column 2 of table 1 plate $\left(\theta_{\text {crit }}=13^{\circ}\right.$ Longitudinal and $28^{\circ}$ Shear $)$. 
additional iterative step [10. The first step of the inversion procedure determines an initial estimate of the elastic constants by fitting simulated bulk-wave TOF values onto the experimental TOF measurements. In the second step of the inversion procedure, a phase-induced time-delay is calculated from the previously obtained elastic properties. This process is then repeated until the required convergence value is achieved, adding considerable complexity to the inversion procedure. Especially for thin samples, the effect of the phase-induced time-delay is substantial. Clearly, the properly calculated TOF results at $f_{c} d=0.5$ MHz.mm (corresponding to the square line in figure 1) show that adding a phase delay to the bulk waves at the liquid/solid/liquid interfaces will not suffice to correct the TOF curve, simply because for such low $f_{c} d$ values, the bulk wave approximation does not longer hold.

Aside from bulk wave limitations and/or ad-hoc required modifications, many of the current inversion techniques are based on using a single in-plane sector scan (corresponding to a fixed in-plane (azimuthal) angle $\phi$ ) at a time [2, 5] 7]. In the case of orthotropic media for example, TOF data for at least three in-plane orientations are considered $\left(\phi=0^{\circ}, 90^{\circ}, 45^{\circ}\right)$, and the individual scans are consecutively provided as input for an inversion algorithm to determine the elastic constants. Accurate information on the actual in-plane orientation of the collected data is crucial in order to obtain good inversion results. Small alignment deviations in the set-up can lead to sizable errors on the elastic constants. In reality, however, the symmetry of the composite sample under investigation, and especially the orientation of its main axes of symmetry, is often unknown a priori. Moreover, when the symmetry orientation of the investigated composite is well defined for an intact sample, mechanical loading during operation could induce a substantial change in fiber direction [11, 12. An approach that uses a consecutive treatment of single scan data at fixed individual azimuthal $\phi$-angles therefore lacks the versatility of acquiring and analyzing the required information in samples of arbitrary symmetry.

As an alternative to the above mentioned techniques, the pulsed ultrasonic polar scan (P-UPS) could be considered. Since its initial conception in the 1980s, the P-UPS technique has been considered as a promising tool in the field of non-destructive characterization of materials [13. In this technique, a certain material spot is insonified with an ultrasonic pulse from a wide range of oblique incidence angles $(\theta, \phi)$, ideally covering the complete upper hemisphere. Here, $\phi$ denotes the in-plane (polar) angle with respect to the $x$-direction, and $\theta$ is the incident angle with respect to the zenit. At each incidence angle, the transmitted and/or reflected signals are recorded, from which the amplitude and/or TOF can be calculated. Figure $3 \mathrm{a}$ represents a schematic of the P-UPS method. Mapping a characteristic (amplitude or TOF) of the transmitted or reflected ultrasonic pulse in polar coordinates yields a $\mathrm{P}$ UPS image in which intriguing patterns can be observed. Figures 3b 3c show experimentally recorded transmission amplitude and TOF P-UPS images for a $\left[0^{\circ}\right]_{8} \mathrm{C} / \mathrm{E}$ composite. A typical experiment records and analyzes signals at 1 million incidence angles and takes about 10 minutes. In this paper, we concentrate on the information contained in the TOF polar scans, in which the TOF is defined as the arrival time associated with the maximum amplitude of the transmitted pulse [1]. The stretched views of the P-UPS images in orthogonal directions are a direct consequence of the unidirectional fiber reinforcement of the investigated $\left[0^{\circ}\right]_{8} \mathrm{C} / \mathrm{E}$ composite.

Recently, several interesting results have been obtained and reported on the application of the P-UPS method for the valuation of composites, including, amongst others, damage assessment [14, 15], (visco)elasticity characterization [14, 15] and strain measurement 16. In the characterization studies, the original P-UPS inversion procedure was based on the amplitude P-UPS in transmission (figure $3 \mathrm{~b}$ and incorporated several aspects of the bulk wave approximation [14, 15. In the first step of the inversion, the characteristic contours in the amplitude P-UPS image, expressing the transmission minima, were matched with computed critical bulk wave angles using a genetic optimization procedure. However, this is only an approximate method as the P-UPS transmission minima are more readily related to a change of the energetically dominant wave in the solid, rather than to the exact occurrence of the critical angles. For many solids the effect of this approximation is small, though it was found to be significant, and thus not negligible, for a typical $\left[0^{\circ}\right]_{8} \mathrm{C} / \mathrm{E}$ composite. To remediate this deviance, a second step was added to the inversion procedure which accounts for a more thorough description of the relevant physical phenomena (interference and attenuation) by matching the measured and simulated transmission amplitude as function of the incidence angle for a selection of in-plane $(\phi)$ angles. The two step procedure finally yielded good inversion results for a range of composites [14, 15. However, because of the insensitivity of the characteristic contours in the P-UPS data to the elastic moduli $C_{i 3}$, with $i=1,2,3$, the original amplitude P-UPS inversion procedure [15] only considered part of the orthotropic tensor.

This paper combines the advantages of the P-UPS technique and classical bulk wave TOF methods, resulting in a novel TOF P-UPS inversion procedure for the characterization of orthotropic elasticity. The proposed TOF P-UPS inversion procedure has the following advantages compared to most of the existing techniques:

- the method is fully applicable to the characterization of plates up to orthotropy

- the method can cope with a wide range of frequencythickness values $f_{c} d$

- information on the mechanical symmetry class of the medium is not required

- a priori knowledge about the orientation of the main axes of symmetry is not required 


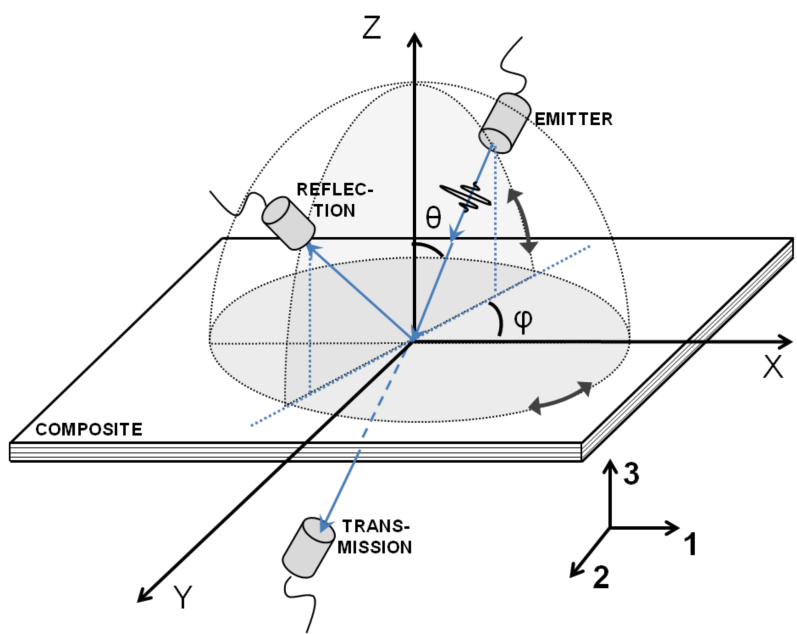

(a)

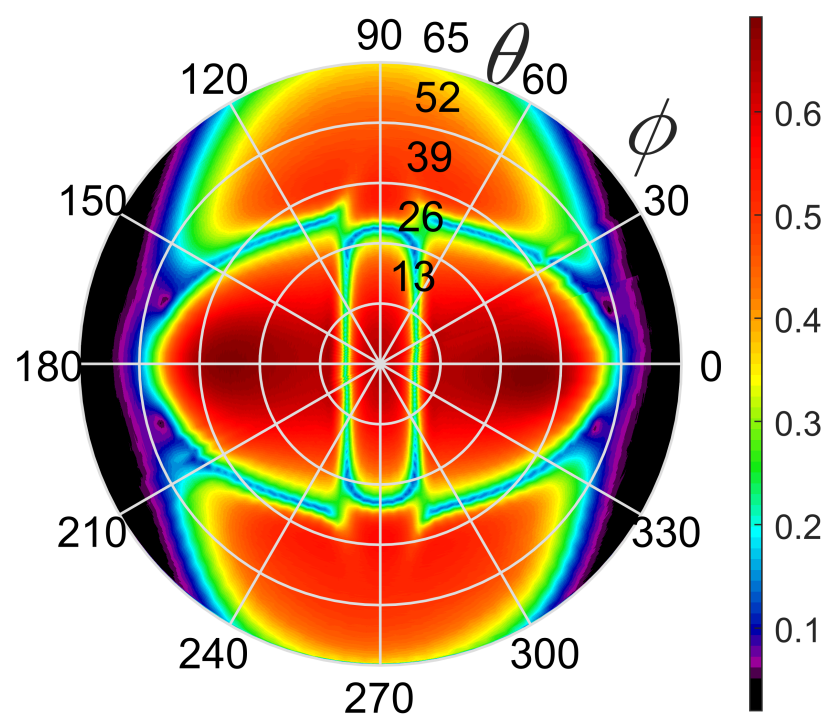

(b)

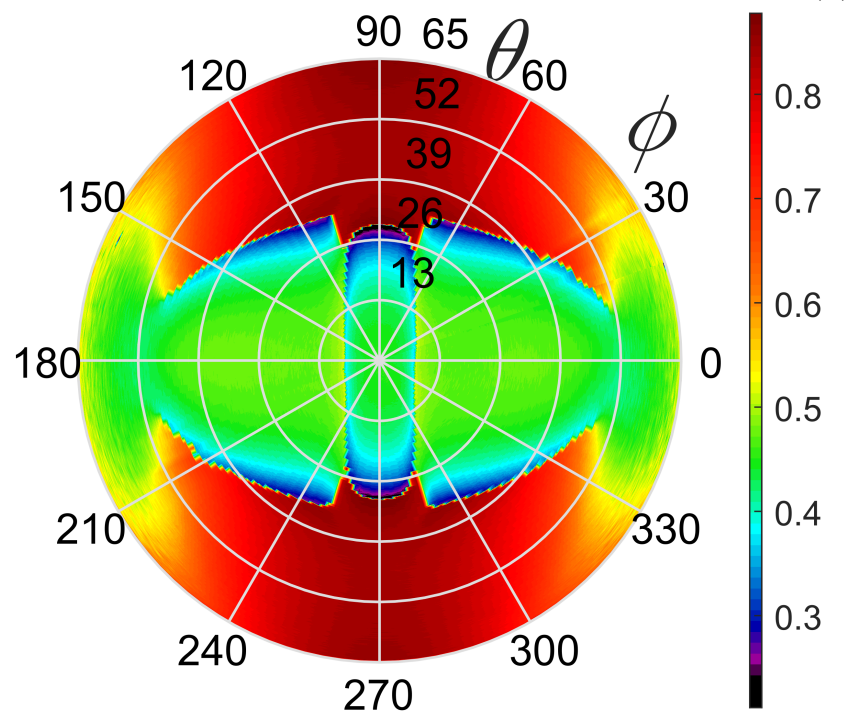

(c)

Figure 3: Schematic of the Pulsed Ultrasonic Polar Scan (P-UPS) principle (a). Transmission P-UPS experiment for a $\left[0^{\circ}\right]_{8} \mathrm{C} / \mathrm{E}$ composite at $f_{c} d=5 \mathrm{MHz} . \mathrm{mm}$ (b)-(c). Amplitude (b) (colorbar in \%). Time-of-flight (c) (colorbar in $\mu \mathrm{s}$ ).

- the method is very robust in a large range of signalto-noise ratios (SNR).

All of the above aspects will be further demonstrated in this paper which is structured as follows. First, the numerical scheme of the TOF P-UPS for forward simulations is presented. Next, the forward model is coupled to an optimization technique on the basis of a genetic algorithm. The inversion procedure is then applied on synthetic data sets representative for an isotropic aluminum and a homogeneous orthotropic C/E composite. For the C/E composite, we consider unidirectional fiber reinforcement, resulting in a material with a high degree of anisotropy, for which we studied the performance of the inversion algorithm under different settings: randomly oriented main symmetry axes, various frequency-thickness $f_{c} d$ values and a range of SNRs. The obtained inversion results are interpreted, discussed, and confronted (where applicable) with inversion data using the traditional bulk wave approximation. Finally, the main conclusions on the novel TOF P-UPS inversion procedure are formulated. For a validation based on experimental data, we refer to a follow-up paper.

\section{Theory: forward model and inversion}

\subsection{Pulsed input signal}

Assuming linear wave propagation, the best way to model the angle dependent transmission through a platelike sample of arbitrary symmetry is to work in the frequency domain. For the TOF P-UPS simulation and in every step of the inversion scheme, the transmitted signal 
can be obtained by multiplying the fourier spectrum of the employed input pulse $I(\omega)$ with the complex valued and frequency dependent transmission coefficient, followed by an inverse fourier transform. In the time domain, this corresponds to a convolution between the input signal $I(t)$ and the impulse response of the material.

In the numerical studies performed in this work we opted to use a pulsed signal of the form

$I(t)=-f_{c} t \cos \left(2 \pi f_{c} t\right) e^{-\left(f_{c} t\right)^{2}}$

where $f_{c}$ represents the center frequency.

The corresponding time domain signal and its frequency spectrum are shown in figure 4. This type of Gaussian damped sine wave is an idealized form of the actual transducer output used in typical ultrasonic testing. The option to work with this realistic signal enables us to easily compare P-UPS experiments with simulations on a qualitative level and to avoid potential instabilities arising from an essential difference between simulated and experimental input signals

\subsection{Transmission coefficient for orthotropic plate-like ma- terials}

In order to determine the transmission coefficient at a particular frequency $f=\frac{\omega}{2 \pi}$ and incidence angle $(\theta, \phi)$, we consider a plane harmonic wave incident on the investigated plate (figure 3a). Inside the material, this harmonic wave will propagate according to the general wave equation for an elastic material with density $\rho$ and elastic constants $C_{i j k l}$ [17, 18]:

$\rho \ddot{u}_{i}=C_{i j k l} \frac{\partial^{2} u_{l}}{\partial x_{j} \partial x_{k}}$

$u_{i}=U_{i} e^{i\left(K_{j} x_{j}-\omega t\right)}$.

Here, $u_{i}$ and $U_{i}$, with $i=1,2,3$, denote respectively the $3 \mathrm{D}$ displacement and polarization components of the wave, and $\left(K_{1}, K_{2}, K_{3}\right)$ the wave vector. As we consider a harmonic plane wave as the impinging signal, it is assumed that the solution of the general wave equation is also plane harmonic in nature. This ultimately leads to an eigenvalue problem known as the Christoffel equation:

$$
\left(C_{i j k l} K_{j} K_{k}-\rho \omega^{2} \delta_{i, l}\right) U_{l}=0 .
$$

Assuming that the $C_{i j k l}$ are rotated over an angle $\phi$ such that the plane of motion is always formed by $\left(x_{1}, x_{3}\right)$ and considering Snell's law (determining $K_{1}$ and $K_{2}$ ), we observe that the condition for a non-zero solution of the Christoffel equation can be generally transformed into a sixth order polynomial in the remaining unknown wavenumber component $K_{3}$ :

$$
\begin{aligned}
\beta_{6} K_{3}^{6}+\beta_{5} K_{3}^{5}+ & \beta_{4} K_{3}^{4}+\ldots \\
& \beta_{3} K_{3}^{3} \beta_{2} K_{3}^{2}+\beta_{1} K_{3}^{1}+\beta_{0}=0 .
\end{aligned}
$$

Using the solutions $K_{3 q}$, with $q=1 . .6$, of equation 6 for the $K_{3}$ component of the wave vector and the corresponding eigenvectors $\left(U_{1 q}, U_{2 q}, U_{3 q}\right)$ for $q=1 . .6$, satisfying equation 5, we are now able to construct a global solution to the wave equation (formula (3)) by considering a linear combination of the six different waves that are allowed to exist in the medium:

$u_{m}=\sum_{q=1}^{6} A_{q} U_{m q} e^{i\left(K_{1} x_{1}+K_{3 q} x_{3}-\omega t\right)} \quad\left\{\begin{array}{l}0 \leqslant x_{3} \leqslant d \\ m=1,2,3 .\end{array}\right.$

However, in most (monoclinic and higher symmetric) materials the coefficients of the odd powers in $K_{3}$ are zero and equation (6) can be reduced to a third order polynomial with only three independent solutions. Considering this reduced form, the linear combination expressed in equation 7 represents a set of three waves going down in the plate and a similar set of waves going up, each of them containing a longitudinal component and two shear components (horizontal and vertical).

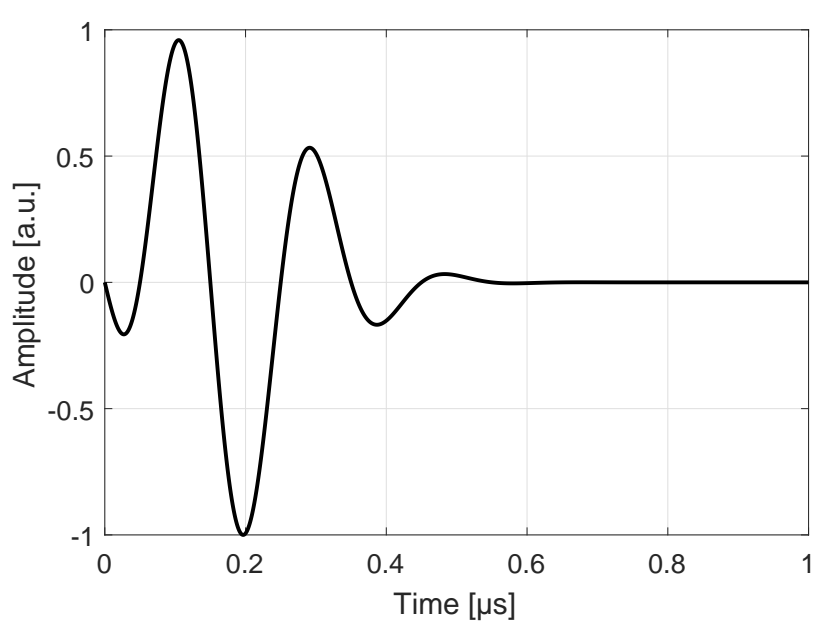

(a) time domain

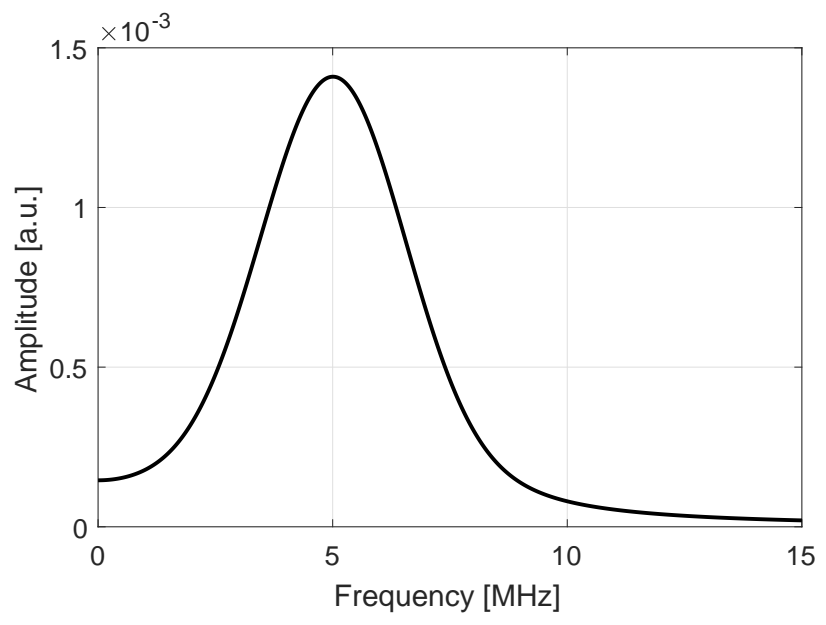

(b) frequency domain

Figure 4: Time domain (a) and frequency domain (b) representation of the input signal given by formula 2 at center frequency $f_{c}=5$ $\mathrm{MHz}$. 
In order to calculate the amplitudes of the individual components, we need to couple the waves in the solid plate to the waves in the loading fluid surrounding the plate, represented by:

$$
\left\{\begin{array}{cl}
u_{m}=\left(A_{I} U_{m I} e^{+}+A_{R} U_{m R} e^{-}\right) & x_{3} \leqslant 0 \\
\text { with } e^{ \pm}=e^{i\left(k_{1} x_{1} \pm k_{3}^{f} x_{3}-\omega t\right)} & m=1,2,3 \\
u_{m}=A_{T} U_{m T} e^{i\left(k_{1} x_{1}+k_{3}^{f}\left(x_{3}-d\right)-\omega t\right)} & x_{3} \geqslant d .
\end{array}\right.
$$

Here, $k_{3 f}$ denotes the $z$-component of the wave vector for the incident wave in the fluid, $\left(U_{1 I}, U_{2 I}, U_{3 I}\right)$ the unit vector along the direction of incidence, $A_{I}$ the amplitude of the incident wave, $\left(U_{1 R}, U_{2 R}, U_{3 R}\right)$ the unit vector along the direction of the reflected wave in the fluid above the sample, $A_{R}$ the amplitude of the reflected wave, and $\left(U_{1 T}\right.$, $\left.U_{2 T}, U_{3 T}\right)$ the unit vector along the direction of the transmitted wave in the fluid below the sample, $A_{T}$ the amplitude of the transmitted wave. By imposing the proper boundary conditions at each side of the plate, i.e. continuity of the normal and tangential stress components $\left(\sigma_{33}\right.$, $\left.\tau_{13}, \tau_{23}\right)$ and of the normal displacement $u_{3}$ :

$$
\begin{cases}\tau_{12}=\tau_{13}=0 & \text { at } x_{3}=0 \text { and } x_{3}=d \\ \sigma_{33} \text { continuous } & \text { at } x_{3}=0 \text { and } x_{3}=d \\ u_{3} \text { continuous } & \text { at } x_{3}=0 \text { and } x_{3}=d .\end{cases}
$$

a system of eight equations in eight unknowns is obtained from which an expression for the scattering coefficients $(R, T)$ in the loading fluid can be found.

\subsection{Transmitted signal and Time-Of-Flight determination}

Next, the transmitted signal in the time domain at an incidence angle $(\theta, \phi)$ is determined by taking the inverse Fourier transform of the input signal $I(\omega)$ multiplied by the transmission coefficient evaluated at the incidence angle $(\theta, \phi)$ for the corresponding frequency component:

$\tilde{T}\left(\theta, \phi, C_{i j k l}, t\right)=\int_{-\infty}^{\infty} I(\omega) T\left(\theta, \phi, C_{i j k l}, \omega\right) e^{i \omega t} \mathrm{~d} \omega$.

Finally, by considering all incidence angles $\left(\phi: 0^{\circ} \rightarrow 180^{\circ}\right.$ and $\theta:-90^{\circ} \rightarrow 90^{\circ}$ ), a TOF P-UPS simulation is obtained in which:

$\operatorname{TOF}\left(\theta, \phi ; C_{i j k l}\right)=\max _{t}\left(\tilde{T}\left(t ; \theta, \phi, C_{i j k l}\right)\right)$

\subsection{Time correction for the experimental Time-Of-Flight Ultrasonic Polar Scan}

Figure 5 shows a TOF P-UPS analysis (cross-section at a fixed $\phi$ angle) from an experiment for an aluminum sample (solid curve) on which we superimposed the numerically determined TOF values following the procedure explained in section 2.3 (square curve). Evidently, there is a systematic error between the TOF prediction and the TOF experiment. The origin of this deviation can be found

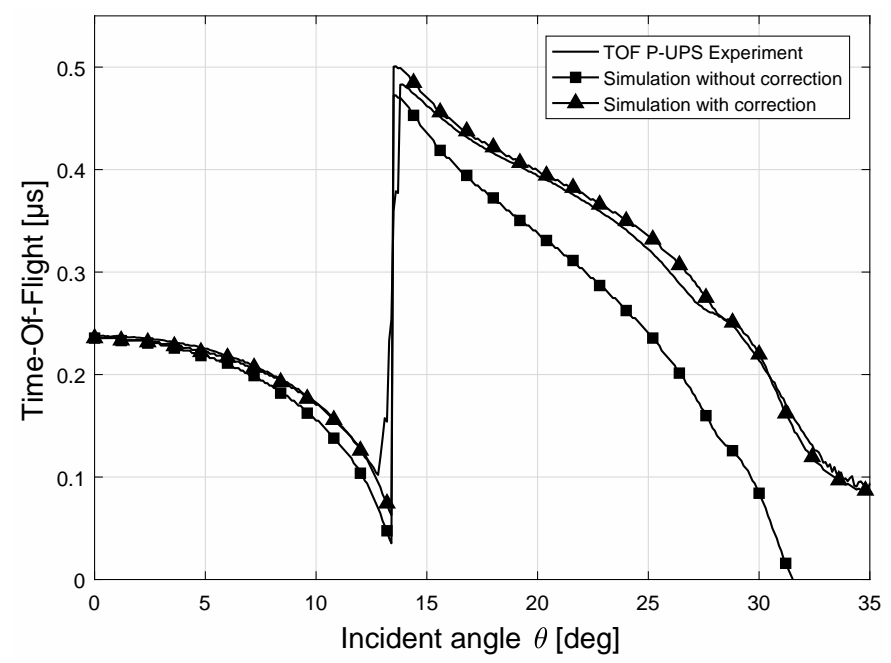

Figure 5: Cross-section ( $\phi$ constant) of a TOF P-UPS experiment at $f_{c} d=5 \mathrm{MHz} . \mathrm{mm}$ for an aluminum plate (solid line) superimposed with simulated TOF-PUPS cross sections (parameters in column 2 of table 1 to illustrate the effect of the correction term accounting for the actual wave emitter-receiver path.

in the difference of the ray path considered in the simulation compared to the actual experiment. Indeed, in the numerical model, the TOF value is calculated as the time stretch between the impingement at $x_{1}=x_{3}=0$ and the arrival of the pulse at $x_{1}=0$ and $x_{3}=d$. In the experiment however, the TOF values are measured by considering the time between departure and arrival of the pulse at the transducers above and beneath the plate. Hence, for a true comparison between an experimental TOF P-UPS and a numerical simulation one should also include the time needed to complete the water path, and we should therefore redefine the numerical TOF P-UPS as the time the wave traveled in the plate plus the time traveled in water. On the one hand, as the polar scan always targets the same point at the surface of the sample, all incident waves travel a distance $R$ above the plate, independent of the incidence angle $\theta$. Beneath the plate, however, we have to take into account that the simulated plane wave leaves the plate at position $(0, d)$ and as such the actual travel distance in the water $D_{f}$ to the receiver position depends on the angle $\theta$ :

$\operatorname{TOF} \operatorname{P-UPS}(R, \theta, \phi)_{\text {num }}=\frac{R}{V_{f}}+\operatorname{TOF}(\theta, \phi)+\frac{D_{f}(R, \theta)}{V_{f}}$.

Figure 6 and some simple trigonometry calculations allow us to express the traveled distance beneath the plate $D_{f}$ according to:

$D_{f}(R, \theta)=R-d \cos \theta$.

As illustrated in figure 5 , the corrected TOF predictions lead to a far better agreement between simulation and experiment, and thus, accounting for the path correction is 


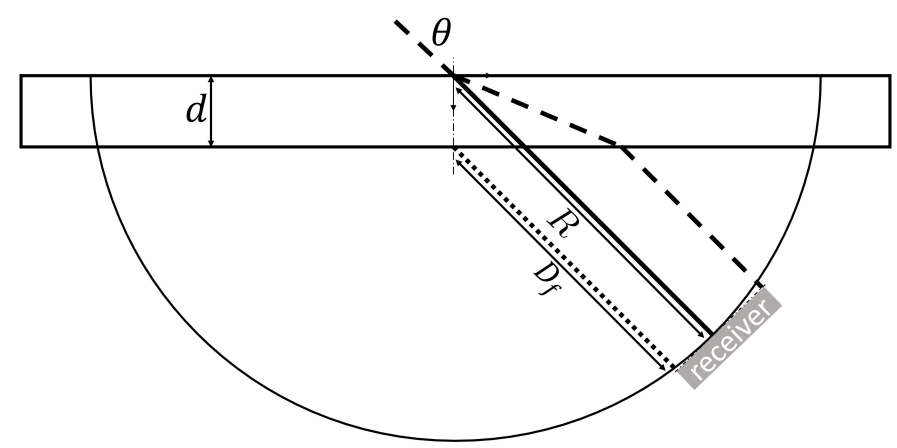

Figure 6: Cross-sectional view of the UPS technique in the plane of wave propagation beneath the plate surface (emitting transducer not shown). The solid line points to the receiver position at an incidence angle $\theta$. The dashed line represents the physical path of a wave incident under angle $\theta$ whilst the dotted line corresponds to the path followed by the simulated transmitted wave. Typical values for the distance between emitting and receiving transducers $(2 R)$ is $120-140$ $\mathrm{mm}$, transducer diameter is around $13 \mathrm{~mm}$ and the plate thickness is in the range of $1-2 \mathrm{~mm}$.

crucial when comparing the TOF P-UPS simulations with experimental data.

\subsection{Inversion scheme}

Finally, the forward model of the TOF P-UPS can now be used in an inversion scheme with the aim to determine the elastic constants $\left(C_{i j k l}\right)$ of the sample under investigation. This is achieved by creating a least square fitness function $\left(F\left(C_{i j k l}\right)\right)$ over different incidence angle combinations $(\theta, \phi)$ :

$F\left(C_{i j k l}\right)=\sum_{\theta, \phi}\left(\operatorname{TOF}_{\exp }(\theta, \phi)-\operatorname{TOF}_{\text {num }}\left(C_{i j k l}, \theta, \phi\right)\right)^{2}$

The fitness function is subsequently minimized by means of a genetic algorithm (GA), in a similar manner as has already been successfully deployed for the determination of mechanical properties from wave velocity measurements [19, 20.

The GA succeeds in this minimization by following the scheme of figure 7a. Starting from an initial generation of 150 sets of $C_{i j k l}$ values, the algorithm determines at every iteration which individuals (sets of $C_{i j k l}$ ) are the 'fittest'(lowest value for the fitness function) in the current generation. The superior $C_{i j k l}$ sets are then used to create descendants, by means of cross-over, mutation and elitism. A new generation, i.e. another 150 sets of $C_{i j k l}$, is composed which is subsequently fed into the next iteration. Repeating this process, the algorithm gradually converges to a generation which consists of superior individuals $C_{i j k l}$. During this iterative procedure, the evolutionary process of the genetic algorithm is stopped when the required level of fitness is achieved or when the maximum amount of iterations (100) are exceeded. Note that the latter only applies in some exceptional cases as most of the time convergence was reached within 40 generations. A typical progress of the fitness function (for the best individual in each consecutive generation) during the optimization process is shown in figure $7 \mathrm{~b}$. As the genetic algorithm is a non-deterministic optimization method, i.e. different initial sets may give different optimization results, the full inversion procedure has been repeated multiple times. For each of the presented cases, typically 100 to 200 repeated inversions were considered from which relevant statistics were extracted (mean and standard deviation). Different normality checks have been performed (Kolgomorogov ( $p$ $=0.5)$, Liliefors $(\mathrm{p}=0.1)$, Anderson-Darling $(\mathrm{p}=0.9)$, Shapiro-Wilk $(\mathrm{p}=0.06))$ on the optimization results, affirming their normal distribution. For instance, figure $7 \mathrm{c}$ shows a typical distribution for $C_{11}$ (note that we will use the Voigt notation $C_{m n}$ for the elastic constants $C_{i j k l}$ from now on 21]) hereafter performing the optimization procedure 200 times. It has been verified that other $C_{i j}$ 's are also distributed normally.

\section{Results and discussion}

In this section, the inversion scheme is applied on different synthetic experiments which are the results of forward TOF P-UPS simulations following the procedure described in section 2.1 2.3. In all cases, the ultrasonic broadband pulse displayed in figure 4 with a center frequency $f_{c}=5$ $\mathrm{MHz}$ is considered, unless specified differently. We assume a temporal grid between 0 and $10 \mu$ s with a resolution $\Delta t$ of $0.025 \mu \mathrm{s}$, corresponding to a resolution $\Delta f$ of $100 \mathrm{kHz}$ in frequency domain. In order to improve the computational efficiency, only a discrete set of the most dominant frequencies in the spectrum of the input signal are used. Typically, 100 to 200 frequency bins, instead of 2000, are considered non-zero in the inverse Fourier transform. It has been verified that the loss of accuracy in the simulation by implementing this restriction is marginal, while the computational efficiency increases by a factor $5-10$. The angular $(\theta, \phi)$-grid ranges from $0^{\circ}$ to $75^{\circ}$ and from $0^{\circ}$ to $360^{\circ}$, respectively, and is discretized in $\Delta \theta=0.1^{\circ}$ and $\Delta \phi=0.5^{\circ}$. The forward simulation results in a synthetic TOF P-UPS data set which is input as the target data in the inversion procedure. The search bounds of the parameter space during the optimization are chosen to be $\pm 50 \%$ of the input values $C_{i j}$ used in the forward modeling. The TOF P-UPS data from five random but equidistant $\phi$ angles between $0^{\circ}$ and $90^{\circ}$ are simultaneously loaded in the inversion procedure. Furthermore, a different temporal discretization and angular grid is used to calculate the transmitted signals during the inverse operation. Doing so, we make sure that no one-to-one relation exists between the forward and the inverse simulation, and as such the problem of 'the inverse crime' is prevented [22]. Moreover, the reduction of frequency bins, applied during the inversion procedure, further decreases the one-to-one relation between the forward and the backward modeling. For 


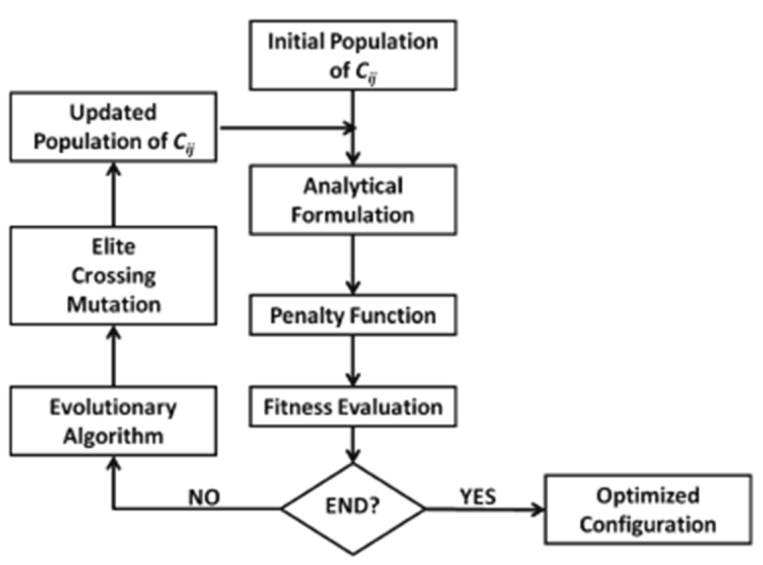

(a)

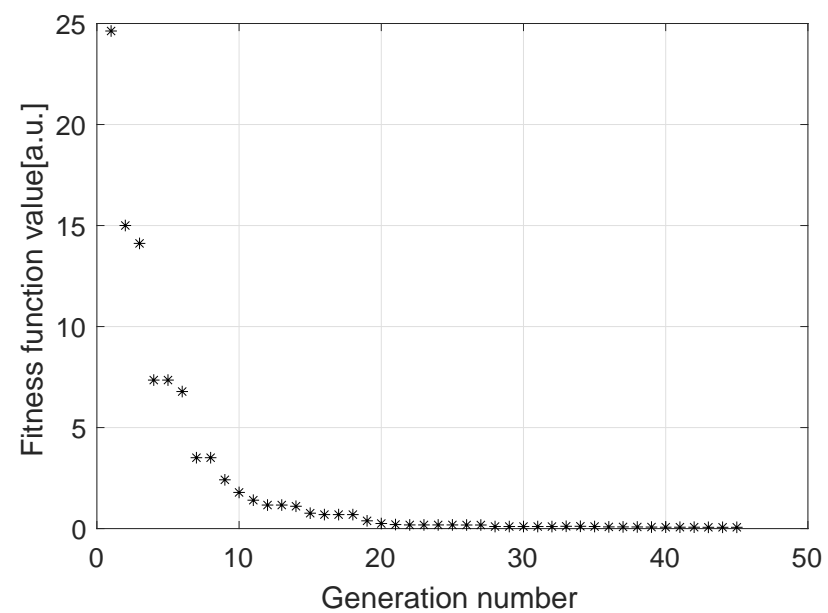

(b)

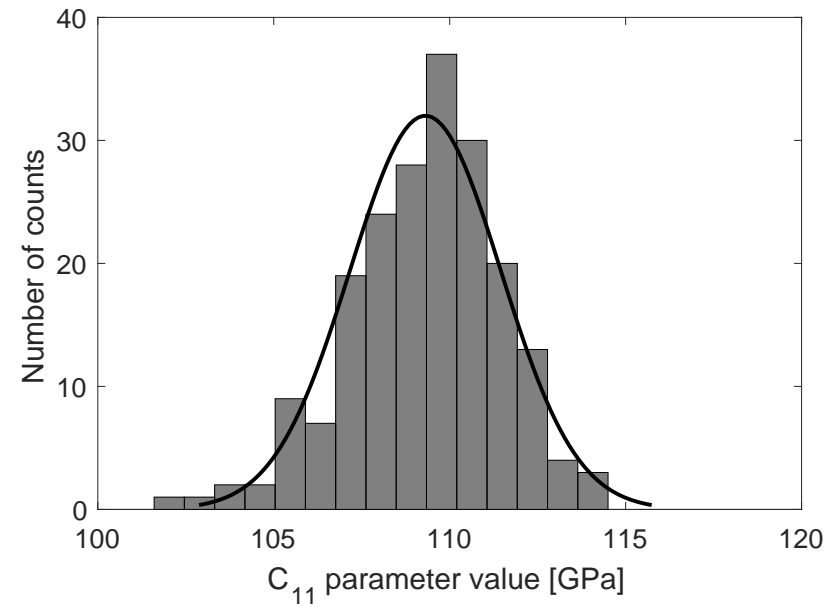

(c)

Figure 7: Schematic of the genetic algorithm inversion procedure (a). Typical convergence of the fitness function during several iterations (b). Histogram of the optimization result considering 200 inversions $\left(C_{11}\right.$ parameter for aluminum TOF P-UPS at $f_{c} d=5 \mathrm{MHz}$.mm), affirming its normal distribution (superimposed black line) (c).

the inversion, an angular grid containing $250 \theta$-points are chosen between 0 and a data dependent maximum. For the temporal domain we opted for a 4096 point grid between 0 and 10ps. Typical run times for one repetition of the inversion procedure are around 2 minutes under optimal computational circumstances using GPU (Tesla K40 NVIDIA). Calculation on CPU resources yields computational times in the order of 30 minutes per inversion. All inversion results, i.e. repetitions using different initial generations, have been used without the removal of possible outliers, unless explicitly specified otherwise. In the following sections, we will illustrate the advantages of the TOF P-UPS method by means of four case studies.

\subsection{Case study I: inversion results for isotropic and or- thotropic plates}

The first example demonstrates the elastic characterization of an isotropic plate, though assuming orthotropic elasticity. A Young's modulus of $70 \mathrm{GPa}$ and a Poisson ratio of 0.34 was considered, which are representative values for aluminum. The plate thickness $d=1 \mathrm{~mm}$ combined with a $5 \mathrm{MHz}$ broadband pulse yields a frequency-thickness value $f_{c} d=5 \mathrm{MHz} . \mathrm{mm}$. The computed TOF P-UPS of the aluminum plate is shown in figure $8 \mathrm{a}$. The circular symmetry of the TOF P-UPS image clearly reveals the isotropic nature of the considered aluminum plate. Although the inversion procedure can significantly be simplified by explicitly taking into account this isotropic symmetry, we assume that this information is unknown prior and during the inversion procedure. Hence, we suppose that the material has an orthotropic symmetry for which 9 independent elasticity constants are required. The results of the inversion procedure, i.e. the mean and standard deviation over 200 runs, are listed in table 2. A good agreement is obtained between the input values and the optimization results, with relative errors $(\Delta)$ in the order of 0 to $1 \%$. The inverted $C_{i j}$ values and their mutual relations $\left(C_{11}=C_{22}=C_{33}=\lambda+2 \mu ; C_{12}=C_{13}=C_{23}=\right.$ $\lambda ; C_{44}=C_{55}=C_{66}=\mu$ ) obviously indicate that the 
Table 2: Inversion results for a numerical aluminum TOF P-UPS simulation at $f_{c} d=5 \mathrm{MHz} . \mathrm{mm}$. The inverted values of the elasticity constants were obtained after 200 inversions.

\begin{tabular}{lccc}
\hline & actual $C_{i j}[\mathrm{GPa}]$ & inverted $C_{i j}[\mathrm{GPa}]$ & $\Delta[\%]$ \\
\hline$C_{11}$ & 110.50 & $109.31 \pm 2.14$ & 1.08 \\
$C_{12}$ & 58.50 & $58.04 \pm 3.49$ & 0.79 \\
$C_{13}$ & 58.50 & $58.39 \pm 2.44$ & 0.19 \\
$C_{22}$ & 110.50 & $109.59 \pm 2.06$ & 0.82 \\
$C_{23}$ & 58.50 & $58.45 \pm 2.37$ & 0.09 \\
$C_{33}$ & 110.50 & $110.82 \pm 3.84$ & 0.29 \\
$C_{44}$ & 26.00 & $25.74 \pm 1.51$ & 0.99 \\
$C_{55}$ & 26.00 & $25.69 \pm 1.51$ & 1.21 \\
$C_{66}$ & 26.00 & $25.78 \pm 1.66$ & 0.48 \\
\hline
\end{tabular}

Table 3: Inversion results for a numerical orthotropic carbon/epoxy TOF P-UPS simulation at $f_{c} d=5 \mathrm{MHz} . \mathrm{mm}$. The inverted values of the elasticity constants were obtained after 200 inversions.

\begin{tabular}{cccc}
\hline & actual $C_{i j}[\mathrm{GPa}]$ & inverted $C_{i j}[\mathrm{GPa}]$ & $\Delta[\%]$ \\
\hline$C_{11}$ & 122.73 & $124.72 \pm 6.85$ & 1.63 \\
$C_{12}$ & 6.57 & $6.47 \pm 0.48$ & 1.35 \\
$C_{13}$ & 6.57 & $6.51 \pm 0.40$ & 0.85 \\
$C_{22}$ & 13.47 & $13.47 \pm 0.33$ & 0.04 \\
$C_{23}$ & 6.55 & $6.58 \pm 0.29$ & 0.44 \\
$C_{33}$ & 13.47 & $13.60 \pm 0.45$ & 0.98 \\
$C_{44}$ & 3.40 & $3.37 \pm 0.21$ & 0.95 \\
$C_{55}$ & 5.86 & $5.79 \pm 0.25$ & 1.18 \\
$C_{66}$ & 6.25 & $6.26 \pm 0.14$ & 0.13 \\
\hline
\end{tabular}

investigated material indeed corresponds to the isotropic symmetry class. This is further confirmed by the Zener anisotropy ratio $Z=\frac{2 C_{44}}{C_{11}-C_{12}}=1.00 \pm 0.10$ which should be equal to 1 for pure isotropy. Hence, these results prove that the TOF P-UPS inversion procedure does not require a priori information about the symmetry class in order to achieve good inversion results.

The second example concerns the determination of the elasticity constants for a material which is representative for a unidirectional $\left[0^{\circ}\right]_{8} \mathrm{C} / \mathrm{E}$ composite (see table 3). The polar image corresponding to the forward TOF P-UPS is displayed in figure $8 \mathrm{~b}$. The results of the inversion for this orthotropic $\mathrm{C} / \mathrm{E}$ composite can be found in table 3. Similar to the previous case, the inversion procedure leads to optimization results which are in close agreement with the actual elasticity parameters. Hence, as a conclusion of this first study, we can safely state that the TOF P-UPS inversion procedure is able to equally cope with media having a low weight as well as a high degree of orthotropy. Good inversion results have been obtained in both cases by considering only minor assumptions (orthotropic symmetry).

\subsection{Case study II: inversion results for an orthotropic plate with a priori unknown symmetry orientation}

The second case study is a direct extension of the previous one. We again consider the $\left[0^{\circ}\right]_{8} \mathrm{C} / \mathrm{E}$ material, but now with a random orientation of the (in-plane) main symmetry axes (rotation over an a priori unknown polar angle). The importance of this case cannot be underestimated as in practical and realistic applications the fiber direction is often unknown. This is especially true for composite materials which show a distortion of the fiber orientation as a consequence of mechanical loading, potentially resulting in a shift of the main symmetry axes [11, 12. Figure $8 \mathrm{c}$ shows a TOF P-UPS simulation of a $\left[0^{\circ}\right]_{8} \mathrm{C} / \mathrm{E}$ composite plate at $f_{c} d=5 \mathrm{MHz} . \mathrm{mm}$, for a random orientation of the main symmetry axes. The orientation of the main symmetry axes with respect to the coordinate system of the TOF P-UPS image is parameterized by means of the deviation angle $\phi_{e}$. Inversions to determine the elastic constants $C_{i j}$ and the deviation angle simultaneously have been performed for the above introduced $\mathrm{C} / \mathrm{E}$ composite, considering deviation angles $\phi_{e}$ up to $20^{\circ}$ in the input data. The deviation angle inversion bounds have been set to $\phi_{e} \in\left[-80^{\circ} ; 80^{\circ}\right]$.

The relative error on the optimized $C_{i j}$ values are visualized in figure 10a as a function of the input value of the deviation angle $\phi_{e}$. Introduction of the new parameter $\phi_{e}$ in the inversion obviously adds complexity to the inversion procedure. Comparison of the results at $\phi_{e}=0$ in figure $10 \mathrm{a}\left(\phi_{e}\right.$ inversion incorporated $)$ with the inversion results in table 3 ( $\phi_{e}$ not incorporated) however, indicates that extending the parameter space of the inversion procedure with an additional unknown $\phi_{e}$ has minor influence on its performance: the obtained errors are still within $1 \%$, averaged over all nine $C_{i j}$ constants. More interesting is the situation where $\phi_{e} \neq 0$. Figure $10 \mathrm{a}$ shows that the relative error on the $C_{i j}$ values remains more or less constant for the different input values of $\phi_{e}$, which is obviously linked to the excellent inversion results for the angle $\phi_{e}$, as listed in column 2 of table 4 .

The larger deviation and uncertainty of the inversion for $\phi_{e}=20^{\circ}$ is potentially an artifact of the very wide parameter space $\left( \pm 80^{\circ}\right)$ of $\phi_{e}$, which may result in the existence of two equally valid solutions $\left(\left(\phi_{e}^{\prime}, C_{i j k l}^{\prime}\right)\right.$ and $\left.\left(\phi_{e}^{\prime \prime}, C_{i j k l}^{\prime \prime}\right)\right)$. Figure 9 illustrates the equivalence between rotating the material coordinate system $\left(x_{1}, x_{2}\right)$ clockwise or counterclockwise. This degeneracy adds complexity in obtaining accurate inversion results, and will definitely yield higher uncertainties. Interestingly, narrowing the bounds for $\phi_{e}$ to $\pm 45^{\circ}$ will prevent the degeneracy, without compromising the inversion procedure under the assumption of orthotropic symmetry.

Note however, that conventional inversion schemes rely on input data at certain well-defined in-plane orientations (e.g. $0^{\circ}, 45^{\circ}$ and $90^{\circ}$ ) with respect to the main symmetry axis, and as such will definitely yield erroneous optimization results if the input data is taken at incorrect in-plane orientations. Even for small deviations in the orientation of the main symmetry axes, considerable errors may be expected for composites with one dominant fiber direction. To quantify the level of the expected errors, the TOF PUPS data for the $\mathrm{C} / \mathrm{E}$ composite at various misalignment 


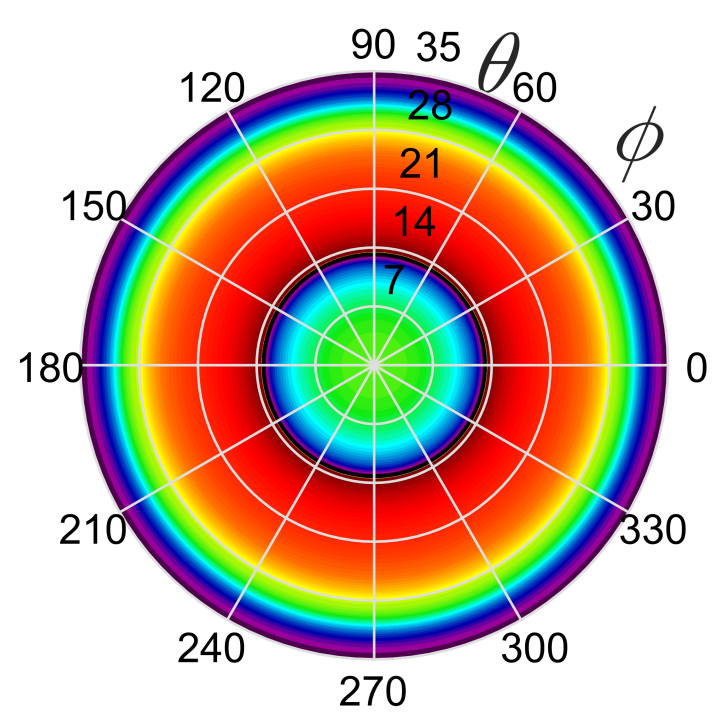

(a)
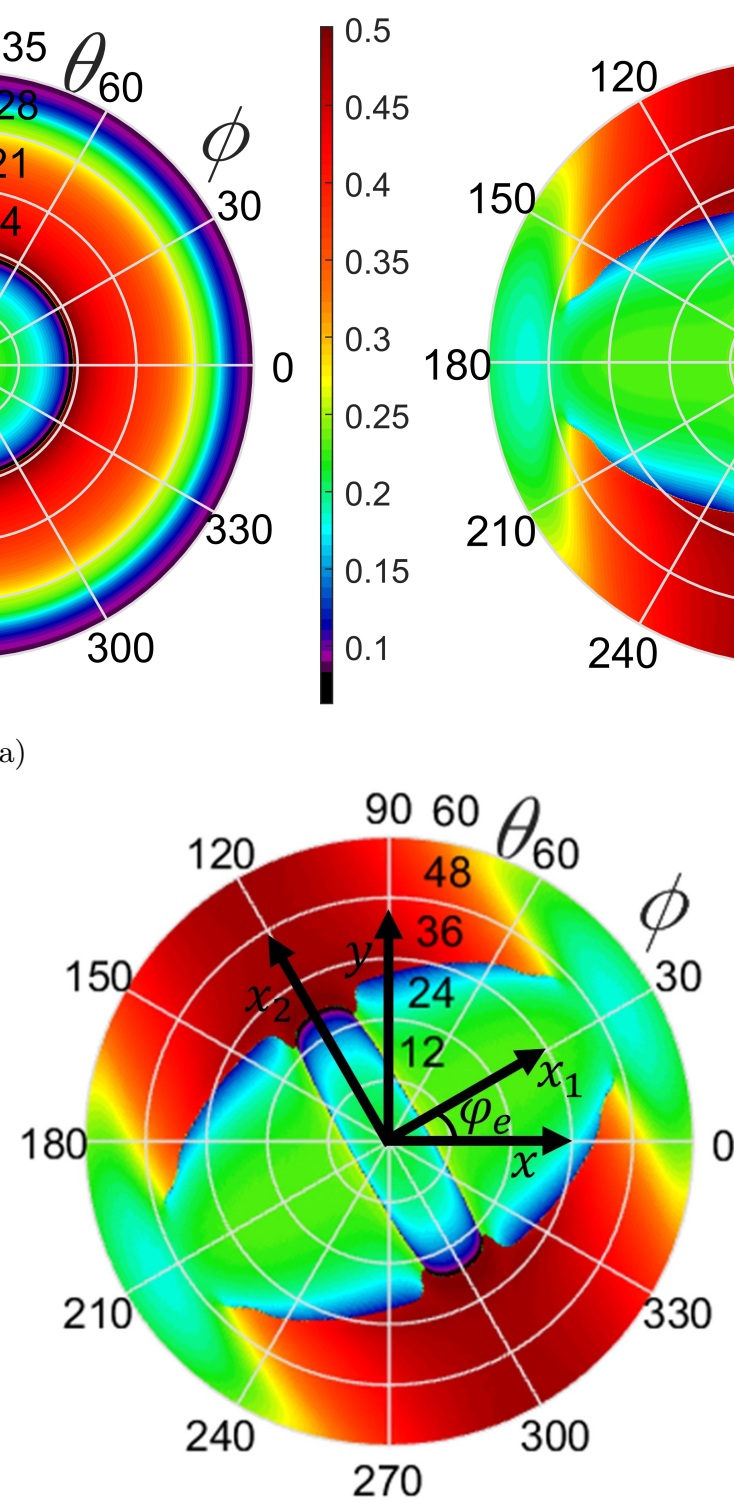

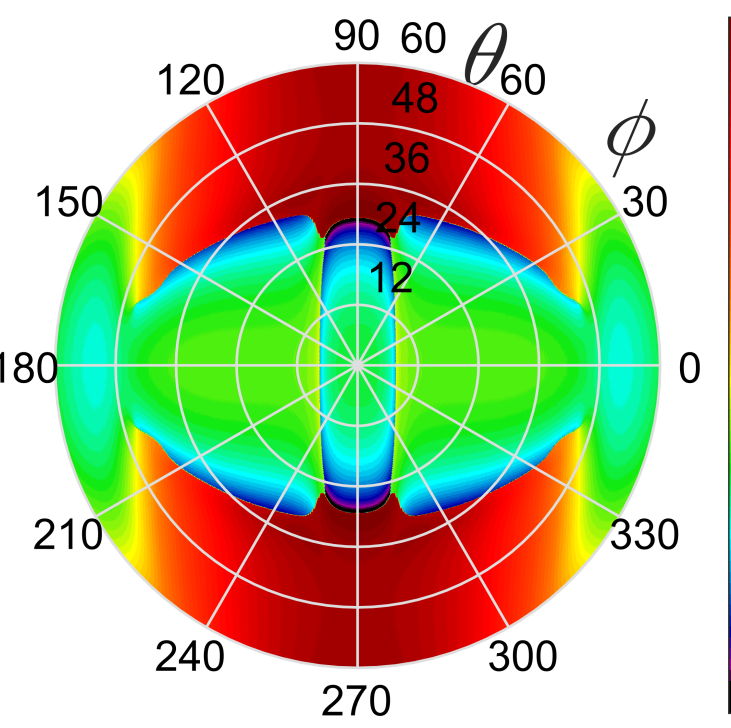

(b)

(c)

Figure 8: Synthetic TOF P-UPS image for an isotropic aluminum plate $\left(f_{c} d=5 \mathrm{MHz} \cdot \mathrm{mm}\right)(\mathrm{a})$ and for an orthotropic $\left[0^{\circ}\right]_{8} \mathrm{C} / \mathrm{E}$ composite $\left(f_{c} d=5 \mathrm{MHz} . \mathrm{mm}\right)(\mathrm{b})$. The corresponding input material parameters are listed in table 1 Subfigure (c) displays the synthetic TOF P-UPS image of the same $\left[0^{\circ}\right]_{8} \mathrm{C} / \mathrm{E}$ composite as in subfigure (b) apart from the fact that the fiber direction has been rotated over an angle $\phi_{e}$ with respect to the intended $0^{\circ}$ direction. The black axes indicate the misalignment between the experimental frame $(x, y)$ and the material frame $\left(x_{1}, x_{2}\right)(\mathrm{c})$.

angles $\phi_{e}$ were again introduced in the inversion routine, but now the inversion algorithm was run by incorrectly assuming that $\phi_{e}=0^{\circ}$. The results of the relative error on the elastic constants after inversion are displayed in figure $10 \mathrm{~b}$ and can be compared in a straightforward way with the results in figure 10 a (be it that the vertical scale is different). The errors for small deviation angles $\phi_{e}$ remain limited, however, once the misalignment angle $\phi_{e}$ exceeds $5^{\circ}$, the deviation on the different $C_{i j}$ quickly rises, leading to poor inversion results. Also, the corresponding standard deviations were observed to rise exponentially (not shown here) in case the deviation angle $\phi_{e}$ was not properly taken into account. This obviously suggests that there are multiple non-ideal solutions across the search region, causing poor convergence of the genetic algorithm.

The results of the second case study thus clearly illustrate that an extra parameter linked to the orientation of the main symmetry axes should be incorporated in any inversion procedure in order to get reliable and accurate results. For an inversion procedure which relies on ultrasonic data obtained at three well-defined angles $\phi\left(0^{\circ}, 45^{\circ}, 90^{\circ}\right)$ with respect to the a-priori assumed position of the main symmetry axis and which processes these three sets of data in a consecutive manner, this may not be straightforward at all. 


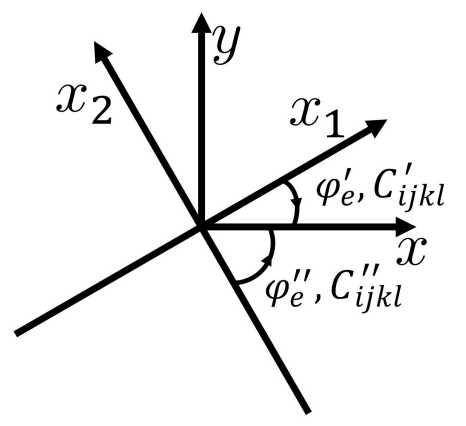

Figure 9: Schematics of the two equally valid solutions $\left(\left(\phi_{e}^{\prime}, C_{i j k l}^{\prime}\right)\right.$ and $\left.\left(\phi_{e}^{\prime \prime}, C_{i j k l}^{\prime \prime}\right)\right)$ which can be achieved by coinciding respectively the $x_{1}$ or $x_{2}$ material symmetry axis with the experimental zero degree in-plane direction ( $x$-axis)

\subsection{Case study III: inversion results for an orthotropic plate with small $f_{c} d$ values}

In the introduction, it was mentioned that small $f_{c} d$ values could become problematic for bulk wave based inversion procedures as the assumption of bulk wave propagation does not longer strictly hold at low frequency or small thicknesses [1, 4, 7, 8]. This was already visually demonstrated in figure 1, and can be easily explained by the fact that a short pulse with higher frequencies (or a plate with large thickness) yields an easy separation in temporal domain of the different (secondary) transmitted pulses. Hence, the TOF of the transmitted signal then simply corresponds to the TOF of the most energetic wave component in the solid. Considering that many realistic (composite) components are plate-like with thin sections, this no-interference condition cannot always be satisfied.

To illustrate this, figures $11 \mathrm{a} \mid 11 \mathrm{c}$ display amplitude color plots of the computed transmission signal in the time domain for a $\mathrm{C} / \mathrm{E}$ plate, insonified at a set of arbitrary incidence angles $(\phi, \theta)$, for a range of frequency-thickness values $f_{c} d$. The superimposed black lines represent the TOF values associated to conventional bulk wave propagation for the different components (QL, QSH, QSV), while the white lines in each subfigure correspond to the TOF of the

Table 4: Inversion results for the $\phi_{e}$ misalignment introduced in an artificial orthotropic plate TOF P-UPS simulation at $f_{c}=5 \mathrm{MHz}$. During the statistics, outliers were removed based on the angle boxplot.

\begin{tabular}{ccc}
\hline actual $\phi_{e}\left[^{\circ}\right]$ & inverted $\phi_{e}\left[^{\circ}\right]$ & $\Delta\left[^{\circ}\right]$ \\
\hline 0 & $0.45 \pm 1.28$ & 0.45 \\
1 & $0.97 \pm 1.17$ & 0.03 \\
2 & $1.99 \pm 1.23$ & 0.01 \\
3 & $2.58 \pm 1.28$ & 0.42 \\
5 & $4.61 \pm 1.19$ & 0.39 \\
8 & $7.50 \pm 1.04$ & 0.50 \\
10 & $9.62 \pm 0.90$ & 0.38 \\
15 & $14.52 \pm 0.67$ & 0.47 \\
20 & $19.32 \pm 1.54$ & 0.68 \\
\hline
\end{tabular}

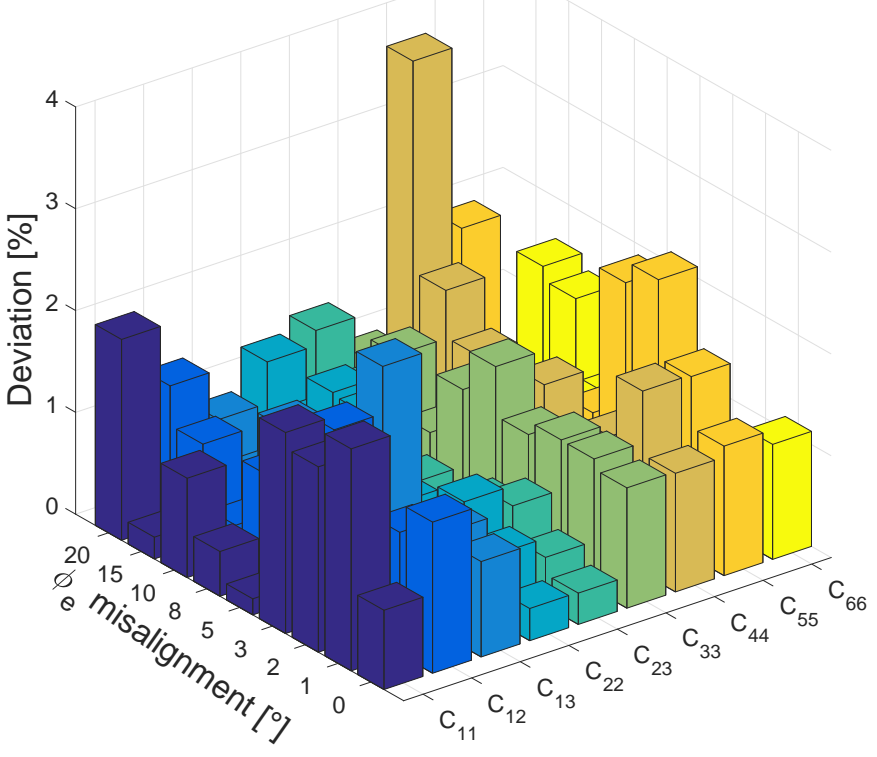

(a)

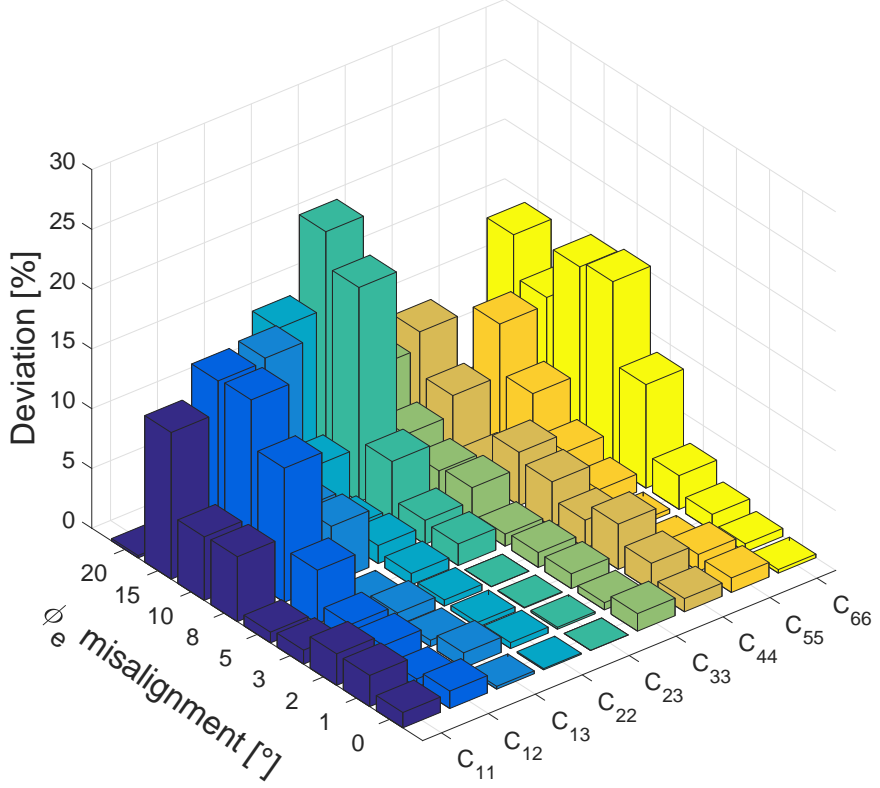

(b)

Figure 10: Deviations of the inverted elastic constants compared to the actual values of an artificial orthotropic plate (column 3 of table 11 with (a) and without (b) taking into account a $\phi_{e}$ misalignment as an additional inversion parameter.

true transmission signal. It can be readily verified that interference of multiple internal echoes and temporal broadening of the pulses leads to a sizeable deviation between both TOF values, especially for small $f_{c} d$ values. Figure 11d shows the relative difference between both TOF profiles for the considered examples $\left(\theta=3,9,25^{\circ}, \phi=40^{\circ}\right)$. For certain incidence angles, the breakdown of the bulk wave approximation starts at a relatively high $f_{c} d$ value (4 MHZ.mm, figure 11b), implying that below this critical value the bulk wave approach will lead to erroneous 


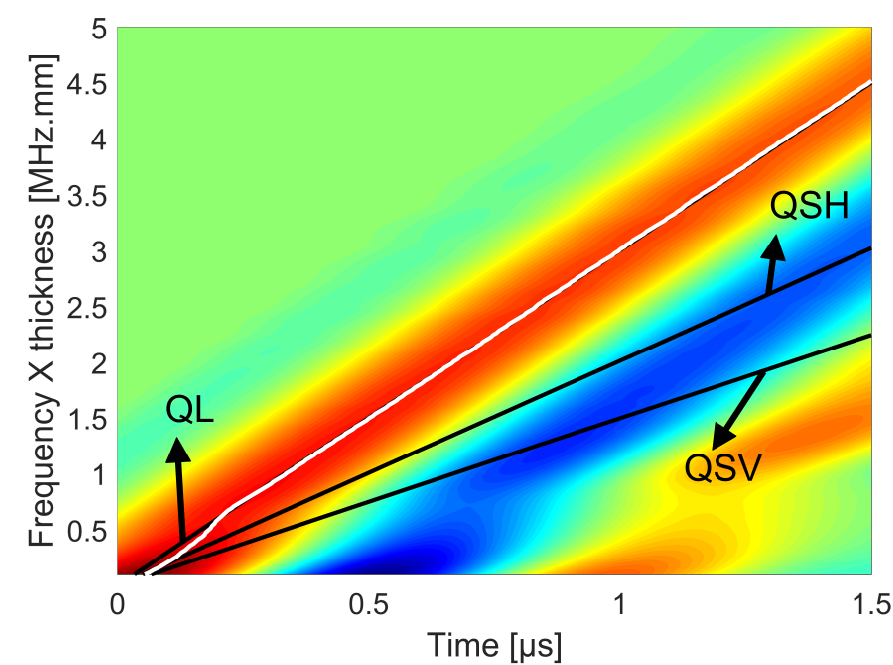

(a) $\theta=3^{\circ}, \phi=40^{\circ}$

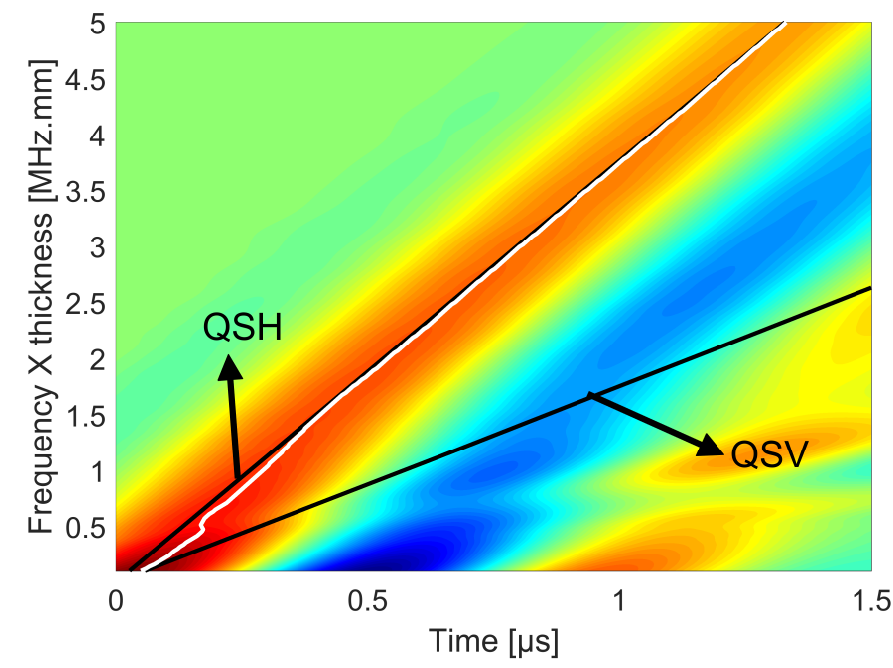

(c) $\theta=25^{\circ}, \phi=40^{\circ}$

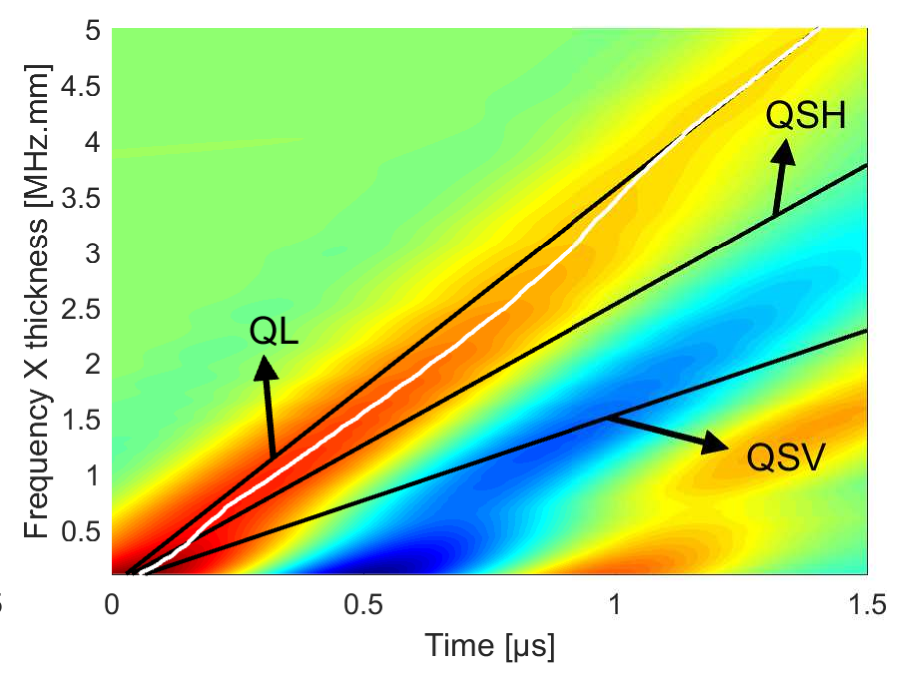

(b) $\theta=9^{\circ}, \phi=40^{\circ}$

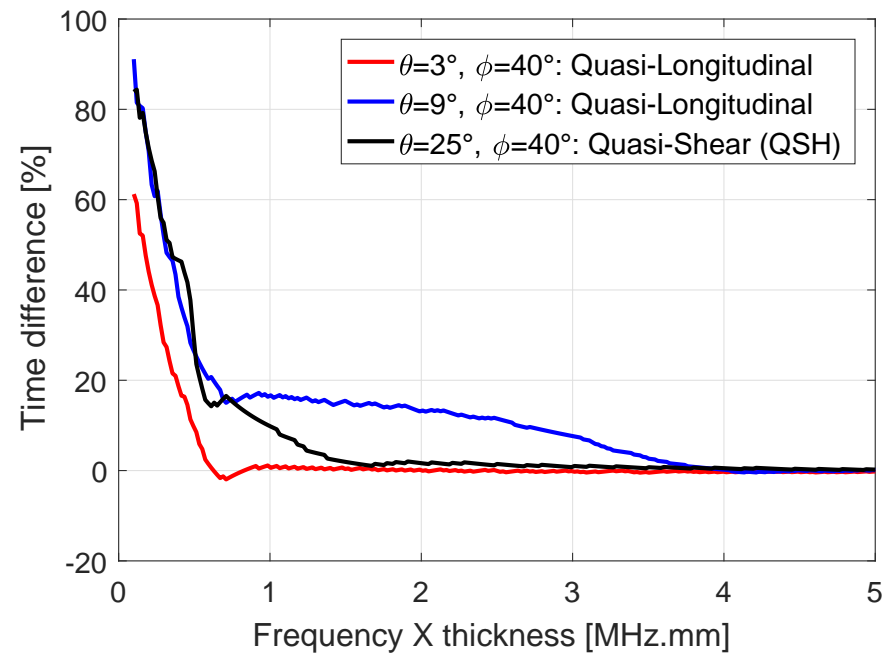

(d)

Figure 11: Simulation of the time signals for an orthotropic plate at different $f_{c} d$ values and various $\theta$ angles $(\mathrm{a})-(\mathrm{c})$. $\phi$ is constant $=40^{\circ}$. The colors depict the amplitude in arbitrary units, red being the highst value and blue the lowest. The black curves represent the bulk TOF of the quasi-longitudinal (QL), quasi-shear horizontal (QSH) and quasi-shear vertical (QSV) components. The white line on the other hand represents the true TOF of the transmitted signal. Subfigure (d) displays the procentual time difference between the TOF of the transmitted pulse and the TOF of the dominant bulk wave as function of frequency $\mathrm{x}$ thickness for the three examples (indicated in the legend).

results, and that there is a need for a more accurate description of the TOF of the transmitted signal. In order to substantiate the opportunity of the TOF P-UPS inversion procedure at low $f_{c} d$ values, we assessed the performance of the inversion routine for a $\left[0^{\circ}\right]_{4} \mathrm{C} / \mathrm{E}$ composite with total thickness $0.5 \mathrm{~mm}$, and an ultrasonic pulse with central frequency $f_{c}=1 \mathrm{MHz}$, yielding a small frequencythickness value $f_{c} d=0.5 \mathrm{MHz} . \mathrm{mm}$. The inversion results are listed in column 3 and 4 of table 5 , and exhibit a very similar good accuracy as the results obtained for the $\mathrm{C} / \mathrm{E}$ at $5 \mathrm{MHz} . \mathrm{mm}$, i.e. relative errors in the range of $0-1 \%$ and small standard deviations. This proves that the proposed TOF P-UPS procedure is thus also fully applicable to plates with small $f_{c} d$ values.

Further, to demonstrate the inappropriateness of bulk wave based inversion schemes for low $f_{c} d$ values, the elas- tic constants of the $\left[0^{\circ}\right]_{4} \mathrm{C} / \mathrm{E}$ composite have also been characterized using the bulk wave approximation. A bulk wave based optimization procedure was implemented employing a genetic algorithm with similar parameters as for the TOF P-UPS inversion procedure. In this way, the potential influence of a numerical bias is mitigated. The inversion results of the bulk-wave approximation approach are summarized in the last two columns of table 5. As expected from a quick analysis of figure 11, the bulk-wave approximation approach leads to large relative errors up to $30 \%$ on the $C_{i j}$ 's.

\subsection{Case study IV: inversion results for an orthotropic plate with low $S N R$}

Finally, the $4^{\text {th }}$ case study explores the robustness of the developed TOF P-UPS inversion procedure to the pres- 
Table 5: Inversion results for a $\left[0^{\circ}\right]_{8} \mathrm{C} / \mathrm{E}$ composite at low $f_{c} d$ values $(0.5 \mathrm{MHz}$.mm). Columns 3 and 4 represent the inversion results obtained with the TOF P-UPS method. The last two columns represent the inversion results obtained with bulk-wave technique.

\begin{tabular}{cccccc}
\hline & & TOF P-UPS & \multicolumn{2}{c}{ Bulk-wave } \\
& actual $C_{i j}[\mathrm{GPa}]$ & inverted $C_{i j}[\mathrm{GPa}]$ & $\Delta[\%]$ & inverted $C_{i j}[\mathrm{GPa}]$ & $\Delta[\%]$ \\
\hline$C_{11}$ & 122.73 & $123.28 \pm 3.99$ & 0.44 & $103.92 \pm 23.10$ & 15.32 \\
$C_{12}$ & 6.57 & $6.55 \pm 0.34$ & 0.18 & $6.86 \pm 1.49$ & 4.48 \\
$C_{13}$ & 6.57 & $6.48 \pm 0.41$ & 1.33 & $6.52 \pm 1.41$ & 0.73 \\
$C_{22}$ & 13.47 & $13.45 \pm 0.16$ & 0.13 & $9.74 \pm 0.98$ & 27.70 \\
$C_{23}$ & 6.55 & $6.53 \pm 0.23$ & 0.34 & $4.37 \pm 0.56$ & 33.32 \\
$C_{33}$ & 13.47 & $13.40 \pm 0.34$ & 0.48 & $12.55 \pm 0.93$ & 6.79 \\
$C_{44}$ & 3.40 & $3.43 \pm 0.16$ & 1.18 & $2.71 \pm 0.52$ & 20.37 \\
$C_{55}$ & 5.86 & $5.85 \pm 0.33$ & 0.22 & $4.77 \pm 0.74$ & 18.64 \\
$C_{66}$ & 6.25 & $6.19 \pm 0.26$ & 0.89 & $6.15 \pm 1.27$ & 1.55 \\
\hline
\end{tabular}
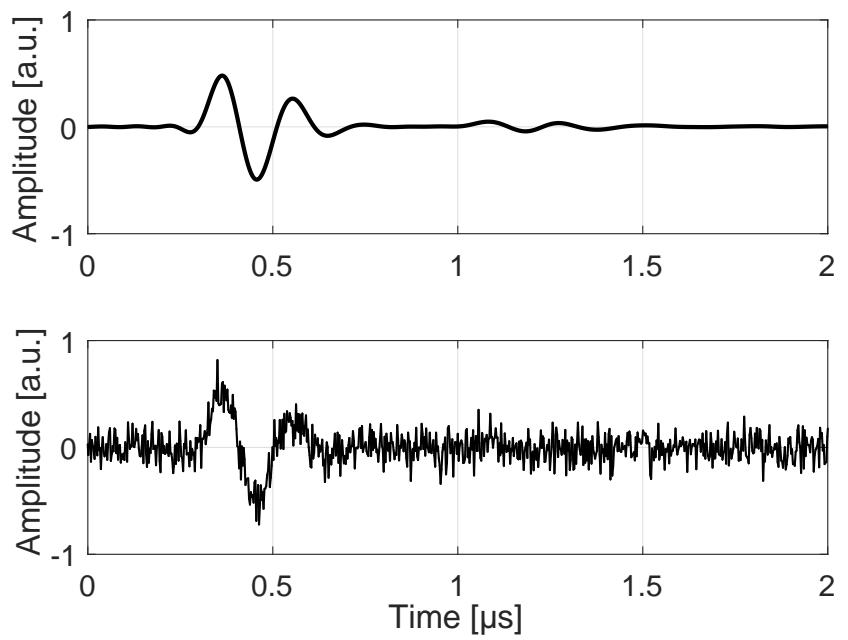

Figure 12: Calculated time signal for a a $\left[0^{\circ}\right]_{4} \mathrm{C} / \mathrm{E}$ composite (material parameters in table 1 at $\theta=5^{\circ}, \phi=40^{\circ}$ : Perfect transmission signal (top) and noisy transmission signal with $\mathrm{SNR}=0 \mathrm{~dB}$ (bottom).

ence of random signal noise. In contrast to synthetic data, real test data will always contain a random scatter component which is likely to reduce the accuracy and/or performance of any inversion procedure. In order to quantify the effect of noise on the inversion method, various levels of scatter have been applied to the forward TOF P-UPS simulation (for a $\mathrm{C} / \mathrm{E}$ sample at $f_{c} d=5 \mathrm{MHz} . \mathrm{mm}$ ) by adding Gaussian white noise on the computed transmission signal in the temporal domain:

$S_{\text {noise }}\left(t_{i}\right)=S_{\text {perfect }}\left(t_{i}\right)+g\left(t_{i}\right) \sqrt{10^{-\frac{\mathrm{SNR}}{10}} P_{\text {input }}}$

where $g\left(t_{i}\right)$ is a random number picked from a standard normal distribution and $P_{\text {input }}$ the power of the input signal. The noise level is defined with respect to the amplitude of the input signal in order to obtain absolute signalto-noise ratios (SNRs).

Different SNR values are considered in this study, starting from a low noise level $(30 \mathrm{~dB})$ to an extremely high

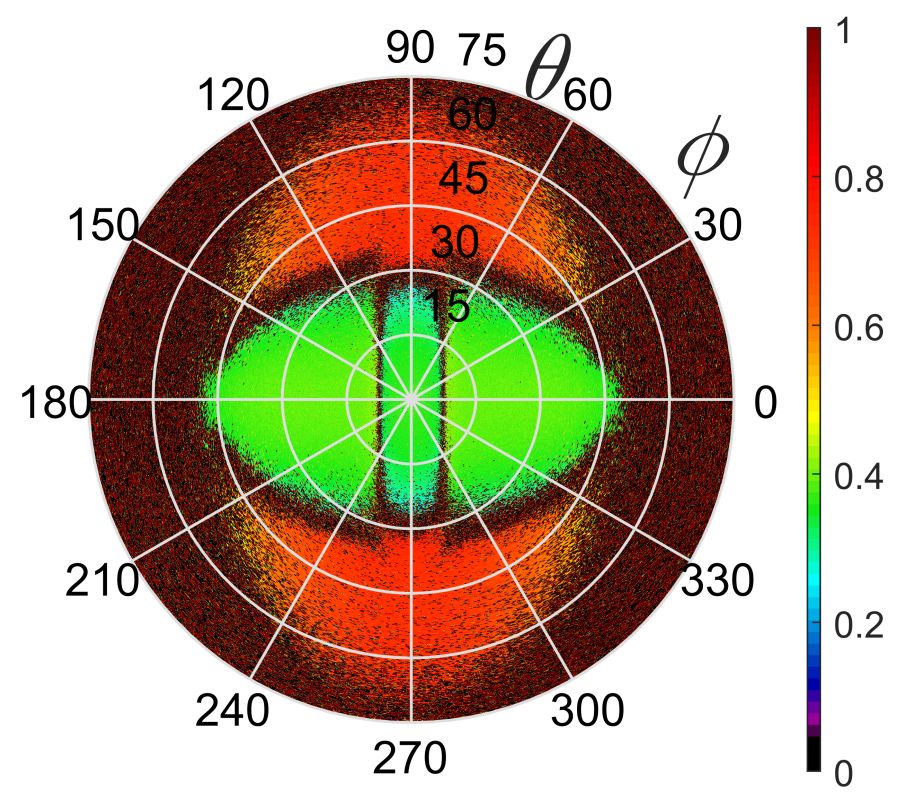

Figure 13: TOF P-UPS for an orthotropic material (table 1) resulting from adding Gaussian white noise $(\mathrm{SNR}=0 \mathrm{~dB})$ on each separate calculated time signal. The input pulse has a center frequency of 5 MHz. The color bar depicts the amplitude in arbitrary units

noise level $(-10 \mathrm{~dB})$. Figure 12 compares the unperturbed and the perturbed transmission signal in the time domain for a SNR of $0 \mathrm{~dB}$ according to Equation (15). Note that, since state-of-the-art P-UPS experiments typically result in a SNR of $\approx 45 \mathrm{~dB}$, a SNR of $0 \mathrm{~dB}$ can be considered as exceptionally bad. The effect of the added noise on the TOF P-UPS simulation is illustrated in figure 13 . The corresponding TOF P-UPS without noise $(\mathrm{SNR}=\infty)$ is shown in figure $8 \mathrm{~b}$. Although the resulting TOF P-UPS depicted in figure 13 exhibits clear scattering, the global geometrical characteristics which are typical for a unidirectional composite are still discernible. This already alludes to the robustness of the TOF P-UPS inversion procedure, even for very low SNRs. The optimization results for the elastic constants in the presence of the different SNR levels are presented in figure 14. High SNRs provide an inversion 


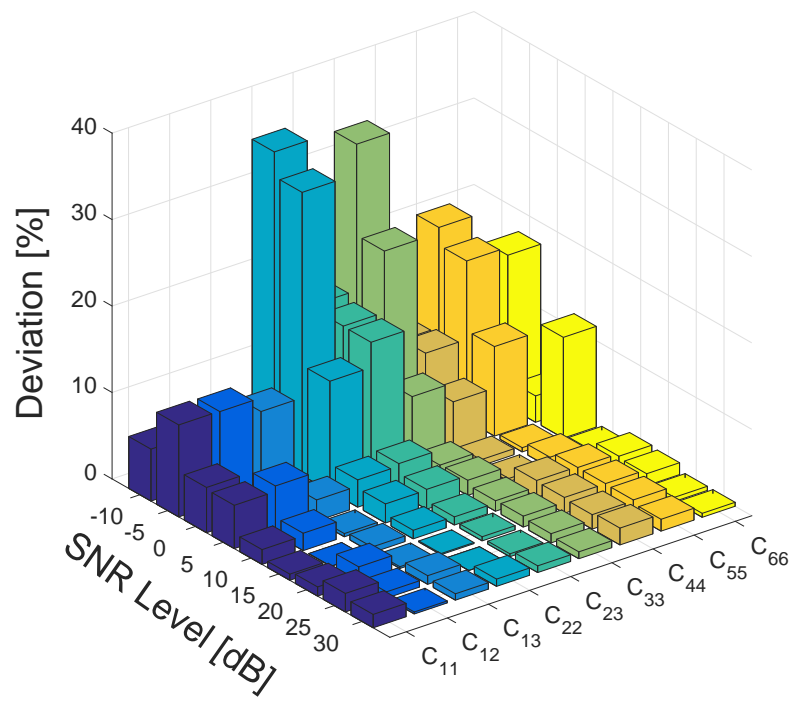

Figure 14: Inversion results for the different elasticity constants of a numerical orthotropic carbon/epoxy TOF P-UPS simulation $\left(f_{c}=5\right.$ $\mathrm{MHz} . \mathrm{mm}$ ) with different levels of artificially added noise.

performance which more or less correspond to the results tabulated in table 3. More interestingly, figure 14 indicates that the quality of the inversion remains stable for SNR levels as low as $10 \mathrm{~dB}$, yielding typical deviations in the range of 0 to $1.5 \%$. Once a SNR of $0 \mathrm{~dB}$ is reached, the inversion error increases significantly to 3 to $10 \%$. Finally, at SNRs smaller than $0 \mathrm{~dB}$, the inversion results in unacceptable errors up to $35 \%$. The stable nature of the TOF P-UPS inversion procedure for noise addition originates from the simultaneous inversion of the TOF data at several $\phi$-angles, and obviously benefits from the fact that it contains a lot of redundant information. A similar observation regarding the robustness to noise and scatter was already made for the amplitude based P-UPS in [14, 15]. Consequently, we can be confident that the P-UPS method has a lot of potential for application in environments where typically lower SNRs are involved.

\section{Conclusion}

This paper introduced an inversion procedure based on the time-of-flight pulsed ultrasonic polar scan (TOF PUPS) for characterizing the elasticity of orthotropic plates. The numerical model of the TOF P-UPS has been presented, and subsequently implemented in an inversion procedure on the basis of a genetic optimization algorithm. The resulting TOF P-UPS inversion procedure has been applied to synthetic data that is representative for a carbon/epoxy composite with a high degree of orthotropy. It is found that the proposed TOF P-UPS inversion procedure yields extremely good inversion results for a range of demanding cases:

- orthotropic media

- a priori unknown symmetry class of the material
- potential angular deviation of the main symmetry axes

- small $f_{c} d$ values

- scattered input data with low SNR.

Explicit comparison between the results obtained with traditional bulk wave based inversion methods and the currently proposed TOF P-UPS inversion procedure indicate the superiority of the TOF P-UPS inversion method. Future efforts will be focused on the combination of amplitude P-UPS and TOF P-UPS data in order to characterize the complex elasticity tensor (including attenuation) of anisotropic plates, and composites in particular.

\section{Acknowledgments}

The research leading to these results has gratefully received funding from the fund for scientific research-Flanders (FWO Vlaanderen, grant 1S45216N and G0B9515N) and the NVIDIA corporation.

\section{REFERENCES}

[1] P.W.A. Stijnman. Determination of the elastic constants of some composites by using ultrasonic velocity measurements. Composites, 26(8):597-604, 1995.

[2] B. Hosten. Reflection and transmission of acoustic plane waves on an immersed orthotropic and viscoelastic solid layer. The Journal of the Acoustical Society of America, 89(6):2745-2752, 1991.

[3] B. Hosten, M. Deschamps, and B.R. Tittmann. Inhomogeneous wave generation and propagation in lossy anisotropic solids. Application to the characterization of viscoelastic composite materials. The Journal of the Acoustical Society of America, 82(5):1763-1770, 1987

[4] S. I. Rokhlin and W. Wang. Double through transmission bulk wave method for ultrasonic phase velocity measurement and determination of elastic constants of composite materials. Acoustical Society of America, 91(6):3303-3312, 1992.

[5] Anna Castellano, Pilade Foti, Aguinaldo Fraddosio, Salvatore Marzano, and Mario Daniele Piccioni. Mechanical characterization of CFRP composites by ultrasonic immersion tests: Experimental and numerical approaches. Composites Part B: Engineering, 66:299-310, 2014.

[6] Victor Munoz, Marianne Perrin, Marie-laetitia Pastor, Hélène Welemane, Arthur Cantarel, and Moussa Karama. Determination of the elastic properties in CFRP composites : comparison of different approaches based on tensile tests and ultrasonic characterization. Advances in Aircraft and Spacecraft Science, 2(3):249-261, 2015

[7] Y. C. Chu, A.D. Degtyar, and S. I Rokhlin. On determination of orthotropic material moduli from ultrasonic velocity data in nonsymmetry planes. The Journal of the Acoustical Society of America, 95(6):3191, 1994.

[8] S. I. Rokhlin and W. Wang. Critical angle measurement of elastic constants in composite material. The Journal of the Acoustical Society of America, 86(5):1876, 1989.

[9] L. Wang and S. I. Rokhlin. Ultrasonic wave interaction with multidirectional composites: modeling and experiment. The Journal of the Acoustical Society of America, 114(5):2582-2595, 2003.

[10] L. Wang, A. I. Lavrentyev, and S. I. Rokhlin. Beam and phase effects in angle-beam-through-transmission method of ultrasonic velocity measurement. The Journal of the Acoustical Society of America, 113(3):1551-1559, 2003. 
[11] Mathias Kersemans, Ives De Baere, Joris Degrieck, Koen Van Den Abeele, Lincy Pyl, Filip Zastavnik, Hugo Sol, and Wim Van Paepegem. Nondestructive damage assessment in fiber reinforced composites with the pulsed ultrasonic polar scan. Polymer Testing, 34:85-96, apr 2014.

[12] M. Kersemans, I. De Baere, J. Degrieck, K. Van Den Abeele, L. Pyl, F. Zastavnik, H. Sol, and W. Van Paepegem. Damage Signature of Fatigued Fabric Reinforced Plastics in the Pulsed Ultrasonic Polar Scan. Experimental Mechanics, 54(8):1467$1477,2014$.

[13] W.H.M. Van Dreumel and J.L. Speijer. Nondestructive composite laminate characterization by means of ultrasonic polar-scan. Materials Evaluation, 39:922-925, 1981.

[14] Mathias Kersemans, Arvid Martens, Joris Degrieck, Koen Van Den Abeele, Steven Delrue, Lincy Pyl, Filip Zastavnik, Hugo Sol, and Wim Van Paepegem. The Ultrasonic Polar Scan for Composite Characterization and Damage Assessment: Past, Present and Future. Applied Sciences, 6(2):58, 2016.

[15] M. Kersemans, A. Martens, N. Lammens, K. Van Den Abeele, J. Degrieck, F. Zastavnik, L. Pyl, H. Sol, and W. Van Paepegem. Identification of the Elastic Properties of Isotropic and Orthotropic Thin-Plate Materials with the Pulsed Ultrasonic Polar Scan. Experimental Mechanics, 54(6):1121-1132, 2014.

[16] Mathias Kersemans, Klaas Allaer, Wim Van Paepegem, Koen Van Den Abeele, Lincy Pyl, Filip Zastavnik, Hugo Sol, and Joris Degrieck. A novel ultrasonic strain gauge for single-sided measurement of a local 3D strain field. Experimental Mechanics, 54(9):1673-1685, 2014.

[17] Adnan H. Nayfeh. Wave Propagation in Layered Anisotropic Media - with Applications to Composites, volume 39 of NorthHolland Series in Applied Mathematics and Mechanics. Elsevier, 1995.

[18] M. Deschamps and C. Chengwei. Reflection/refraction of a solid layer by Debye's series expansion. Ultrasonics, 29(4):288-293, 1991.

[19] Krishnan Balasubramaniam and Navin S. Rao. Inversion of composite material elastic constants from ultrasonic bulk wave phase velocity data using genetic algorithms. Composites Part B: Engineering, 29(2):171-180, 1998.

[20] A. Marzani and L. De Marchi. Characterization of the elastic moduli in composite plates via dispersive guided waves data and genetic algorithms. Journal of Intelligent Material Systems and Structures, 24(17):2135-2147, 2012.

[21] Yakov Itin and Friedrich W. Hehl. The constitutive tensor of linear elasticity: Its decompositions, Cauchy relations, null Lagrangians, and wave propagation. Journal of Mathematical Physics, 54(4):042903, 2013.

[22] Armand Wirgin. The inverse crime. arXiv.org, pages 1-10, 2004. 